\title{
Selective axonal growth of embryonic hippocampal neurons according to topographic features of various sizes and shapes
}

This article was published in the following Dove Press journal:

International Journal of Nanomedicine

21 December 2010

Number of times this article has been viewed

\author{
David Y Fozdar ${ }^{*}$ \\ Jae Y Lee ${ }^{2 *}$ \\ Christine E Schmidt ${ }^{2-6}$ \\ Shaochen Chen ${ }^{1,3-5,7}$ \\ 'Departments of Mechanical \\ Engineering, ${ }^{2}$ Chemical Engineering, \\ ${ }^{3}$ Biomedical Engineering; ${ }^{4}$ Center \\ for Nano Molecular Science and \\ Technology; ${ }^{5}$ Texas Materials \\ Institute; ${ }^{6}$ nstitute of Neuroscience; \\ ${ }^{7}$ Microelectronics Research Center, \\ The University of Texas at Austin, \\ Austin, TX, USA \\ *Contributed equally to this work
}

Correspondence: Christine E Schmidt

Department of Biomedical Engineering,

BME 4.202I, MC C0800, The

University of Texas at Austin,

Austin, TX 787/2-0292, USA

$\mathrm{Tel}+\mathrm{I} 51247 \mid 1690$

Fax + I 5I2 47| 06I6

Email schmidt@che.utexas.edu
Purpose: Understanding how surface features influence the establishment and outgrowth of the axon of developing neurons at the single cell level may aid in designing implantable scaffolds for the regeneration of damaged nerves. Past studies have shown that micropatterned ridgegroove structures not only instigate axon polarization, alignment, and extension, but are also preferred over smooth surfaces and even neurotrophic ligands.

Methods: Here, we performed axonal-outgrowth competition assays using a proprietary four-quadrant topography grid to determine the capacity of various micropatterned topographies to act as stimuli sequestering axon extension. Each topography in the grid consisted of an array of microscale (approximately $2 \mu \mathrm{m}$ ) or submicroscale (approximately $300 \mathrm{~nm}$ ) holes or lines with variable dimensions. Individual rat embryonic hippocampal cells were positioned either between two juxtaposing topographies or at the borders of individual topographies juxtaposing unpatterned smooth surface, cultured for 24 hours, and analyzed with respect to axonal selection using conventional imaging techniques.

Results: Topography was found to influence axon formation and extension relative to smooth surface, and the distance of neurons relative to topography was found to impact whether the topography could serve as an effective cue. Neurons were also found to prefer submicroscale over microscale features and holes over lines for a given feature size.

Conclusion: The results suggest that implementing physical cues of various shapes and sizes on nerve guidance conduits and other advanced biomaterial scaffolds could help stimulate axon regeneration.

Keywords: axon guidance, micropatterning, polarization, surface topography, tissue engineering

\section{Introduction}

Advances in nerve tissue engineering may ultimately lead to new ways of treating neurologic problems and/or diseases by regenerating degraded or necrotic nerve tissue. Much focus is being placed on the development of porous biomaterials that can present combinations of various stimulative extracellular cues, eg, endogenous cells and physical and chemical stimuli, to autologous neural stem cells and immature neurons. ${ }^{1,2}$ These types of advanced biomaterials will be used as support constructs (scaffolds) providing neuronal cells with a realistic microenvironment containing chemical and physical cues that induce, sustain, and enhance tissue development and viability. Moreover, it is likely that a variety of stimulative physical cues will be incorporated onto scaffolds, which will be implemented based on their empirically determined efficacy. Schmidt and Leach ${ }^{3}$ provided a comprehensive review of clinically relevant tissue engineering strategies for the repair and regeneration of damaged nerve tissue. 
Current strategies to repair damaged nerves include suturing nerve endings, implanting autologous nerves, and using nerve guidance conduits. Nerve sutures are useful in repairing damage on a small scale; however, for damage to larger portions of nerve, suturing requires placing a large amount of mechanical (stretching) tension on nerves, which has been shown to inhibit regeneration. Autologous nerve grafts are the standard in terms of treating large-scale nerve damage in patients. In a nerve graft, a nerve is taken from an inconspicuous (ideally speaking) section of a patient's body and reinserted at the point of injury. Unfortunately, this technique is limited by the fact that new damage is often inflicted at the point where the therapeutic nerve was excised. Along with cell-based therapies consisting of the injection of cell suspensions, which has been done, clinically, to a limited degree, transplantations of cells seeded on nerve guidance conduits have been shown to help regenerate damaged tissues. Nerve guidance conduits are simple tubular structures made of polymers that are sutured to the defect site. Nerve guidance conduits aid in guiding growth of axons sprouting from the proximal end of the nerve damage gap, while also providing the damaged nerve with access to various growth facilitating biochemicals. The utilization of nerve guidance conduits to repair nerve damage has been shown to minimize the formation of scar tissue. ${ }^{3}$ Schwann cells isolated from adult nerves were found to stimulate the regeneration of nerves guided in nerve guidance conduits. ${ }^{4}$ Schmidt et $\mathrm{al}^{5}$ were able to stimulate the outgrowth of neurites from damaged nerves by transplanting Schwann cells and applying a small voltage.

At this point, current nerve guidance conduits are limited by the fact that they do not have an architecture that mimics the shape and range of scales inherent to native tissue, thus rendering them insufficient for restoring nerve tissue beyond what has been achieved by conventional autologous nerve grafts. In vivo the extracellular matrix of neurons serves as a mechanical support and introduces various physical and biochemical topography that modulates cell division, migration, adhesion, and axon/neurite formation in immature neurons. Major extracellular matrix components in the peripheral nervous system have chemical (eg, laminin) and physical structures, with microscale and submicroscale dimensions. Thus, biomaterials will require the incorporation of various small scale chemical and physical cues to promote intimate contact that mimics the interactions exploited by neurons in their natural habitat.

Microfabrication techniques have been adopted to pattern surfaces with well ordered features to study the effects of topography on neuronal behavior. ${ }^{6-10}$ Studies on the interactions between microfabricated topographic cues and neurons have revealed the important role of physical cues in inciting a myriad of cellular behaviors, including adhesion, migration, and differentiation. Rajnicek et al ${ }^{11,12}$ performed one of the first indepth studies on the effects of line arrays on the alignment of axons of hippocampal neurons. They showed that topography and its dimensions heavily affect axonal alignment. Gomez et a ${ }^{13}$ performed novel competition axon guidance assays by culturing individual hippocampal neurons between two micropatterned polydimethylsiloxane surfaces, one containing microscale lines and the other various neuroactive biomolecules, such as nerve growth factor and laminin. They found that embryonic hippocampal neurons extended their axons preferentially toward the $2 \mu \mathrm{m}$ line topographies relative to smooth polydimethylsiloxane surfaces or polydimethylsiloxane patterned with nerve growth factor and laminin, emphasizing the relative ability of topography to stimulate axonal growth. To date, there have not been similar competition studies evaluating preferential axonal growth among topographies of varying feature shape and size. Therefore, we investigated the relative abilities of microscale and submicroscale lines and holes to influence axonal guidance by performing novel competitive assays. Immature neurons were micropositioned at select locations around a four-quadrant topography grid consisting of $300 \mathrm{~nm}$ and $2 \mu \mathrm{m}$ holes and lines (four topographies in total, one per quadrant). We investigated the ability of the above topographies to influence axon formation and growth in neurons based on the distance between the neurons and topographies and the shape and size of the features making up the topographies.

\section{Material and methods Quartz substrate fabrication}

Quartz substrates of size $25 \mathrm{~mm}^{2}$ were exposed to oxygen plasma (50 sccm O, 300 Watts, $150 \mathrm{mTorr}, 25^{\circ} \mathrm{C}$; PlasmaTherm 790, Plasma-Therm Inc, St Petersburg, FL) for 10 minutes and immersed in a mixture of $25 \%$ hydrogen peroxide $\left(30 \% \mathrm{H}_{2} \mathrm{O}_{2}\right.$ in water) $(\mathrm{v} / \mathrm{v})$ in sulfuric acid (piranha bath) for 10 minutes (hydrogen peroxide 30\% $\# 2190$, sulfuric acid 96\% \#9684, JT Baker, Phillipsburg, NJ). The substrates were removed from the bath, thoroughly rinsed in deionized water, dried with $\mathrm{N}_{2}$ gas, and dehydrated on a hot plate at $200^{\circ} \mathrm{C}$ for five minutes. After cleaning, a thin $30 \mathrm{~nm}$ layer of chromium was thermally evaporated onto the quartz at a rate of $5 \AA / \mathrm{sec}$ (Explorer, Denton Vacuum, Moorestown, NJ). ZEP520A (Zeon Chemicals, Louisville, KY) positive electronic 
resist was coated onto the chromium layer to a thickness of approximately $200 \mathrm{~nm}$ by spinning at $4000 \mathrm{rpm}$ for 40 seconds; nominal layer thickness was reduced by diluting the ZEP in anisole to a concentration of $50 \%(\mathrm{v} / \mathrm{v})$. After spin-coating, the resist was baked on a hot plate at $180^{\circ} \mathrm{C}$ for 150 seconds.

Arrays of structures were patterned in the ZEP using electron beam lithography (JEOL 6000 FSE, JEOL Ltd, Tokyo, Japan; Raith 50, Raith GmbH, Dortmund, Germany) with a beam fluence of $100 \mu \mathrm{C} / \mathrm{cm}^{2}$ and subsequently developed in ZED-N50 (Zeon Chemicals) by double-spraypuddle for $15+15$ seconds using standard pipettes. Isopropyl alcohol was used as the etch-stop during the developing process. The substrate was dried with a slow stream of $\mathrm{N}_{2}$ gas. The ZEP resist served as a dry-etch mask for the underlying chromium layer, which provided a selectivity close to 3:1 chromium:ZEP. A two-step reactive ion-etching (Trion Technology, Clearwater, FL) process was used to etch through the chromium. The first step was a descum $\mathrm{O}_{2}$ plasma treatment to remove residual resist from developed regions. The second step was the chromium etch step, which was timed to etch completely through the chromium layer to the quartz. The ZEP resist did not have to be stripped after etching through the chromium layer because remaining resist was stripped rather quickly during the following quartz etch. The chromium layer served as the etch mask for the underlying quartz with a selectivity of over 10:1 quartz:chromium. The quartz was etched down about $400 \mathrm{~nm}$. After quartz etching, the remaining chromium was stripped with a chromium wetetchant (Etchant 1020, Transene Company, Danvers, MA) at $40^{\circ} \mathrm{C}$ for two minutes. The quartz was then thoroughly washed in a piranha bath for 10 minutes and stored in distilled water for later experimentation.

\section{Design of topographies}

Topographies consisted of single structures arrayed in either one (lines) or two dimensions (holes) and were strategically chosen based on results obtained by Gomez et $\mathrm{al}^{13}$ and on additional design rules. Gomez et al showed that $2 \mu \mathrm{m}$ lines were more effective at stimulating axon polarization in hippocampal neurons than chemical ligands; they also showed that $1 \mu \mathrm{m}$ lines were more stimulative than $2 \mu \mathrm{m}$ lines. In light of their investigations, we chose to compare $2 \mu \mathrm{m}$ lines with lines having a width of $300 \mathrm{~nm}$, to determine whether an order-of-magnitude decrease in line width would further enhance neuronal responses. Because we also wanted to investigate how different feature shapes would affect axon formation, we decided to compare the lines with circular holes of equivalent dimension (line width $\approx$ diameter) and to compare holes of different diameters. Thus, four topographies were designed and combined to form a four-quadrant competition grid to facilitate data acquisition (Figure 1).

Each quadrant consisted of an array of structures and was separated from neighboring topographies by unpatterned gaps of $20 \mu \mathrm{m}$ width. Each competition scheme was defined as a competition between two juxtaposing topographies vying to sequester the axon of a polarized nerve cell. In this study, topographies included holes with a $2 \mu \mathrm{m}$ diameter with horizontal and vertical spacings of $1 \mu \mathrm{m}$, lines of $2 \mu \mathrm{m}$ width with a spacing of $1 \mu \mathrm{m}$, holes with a $300 \mathrm{~nm}$ diameter with horizontal and vertical spacings of $1 \mu \mathrm{m}$, and lines of $300 \mathrm{~nm}$ width with a spacing of $1 \mu \mathrm{m}$. Throughout the paper, we sometimes refer to the $300 \mathrm{~nm}$ structures as "submicroscale", although structures having dimensions less than $1 \mu \mathrm{m}$ are often called "nano" in bioapplications (as opposed to $100 \mathrm{~nm}$ ).

In our efforts to compare differences in feature shape, we decided to study lines and holes due to some self-imposed design criteria. We define a homogeneous topography as a surface formed when a single structural formation is arrayed over the entire area to be patterned in a single-step with minor to no subsequent modifications. Fundamental structural shapes include groove-ridge (ie, lines) and hole/pillar, which represent structures arrayed in one direction and two directions with a particular pitch (ie, distance between common points among two identical adjacent structures), respectively. According to our definition of homogeneous, combinations of structures or structural gradients based on size would render the resulting topography inhomogeneous. Several variations of the two fundamental shapes of homogeneous topographies (lines and holes) exist, but we believe that those modifications are simply variations of simple lines and holes and do not represent major changes in shape.

The arrays of $300 \mathrm{~nm}$ structures were patterned using a special method that reduced the electron beam writing time (Raith 50 EBL system) significantly, convenient for writing dense structure arrays over relatively large areas, ranging in scale from square micrometers to centimeters. Patterning structures over a large area is a commonplace requirement for patterning dense arrays of submicroscale features (critical dimension $<1 \mu \mathrm{m}$ ) for performing cell studies. Typically, objects are patterned with EBL as closed polygons bounded by a finite number of vertices. While straight edges simply connect two vertices, curved features can consist of a very large number of vertices (a curved edge is essentially the serial aggregate of tiny lines attached end-to-end). Increasing the accuracy of edge curvature requires the specification of 
A
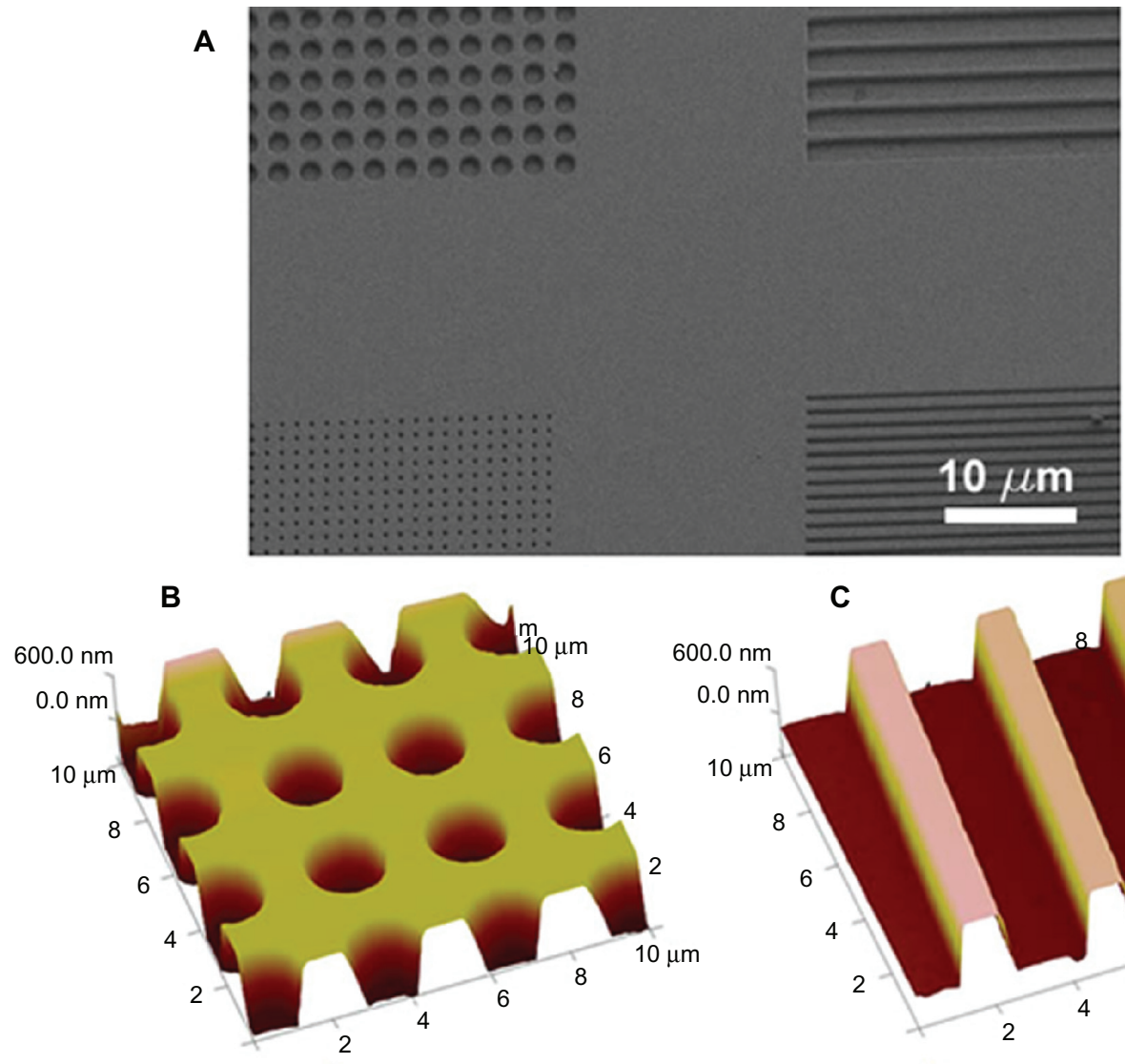

C
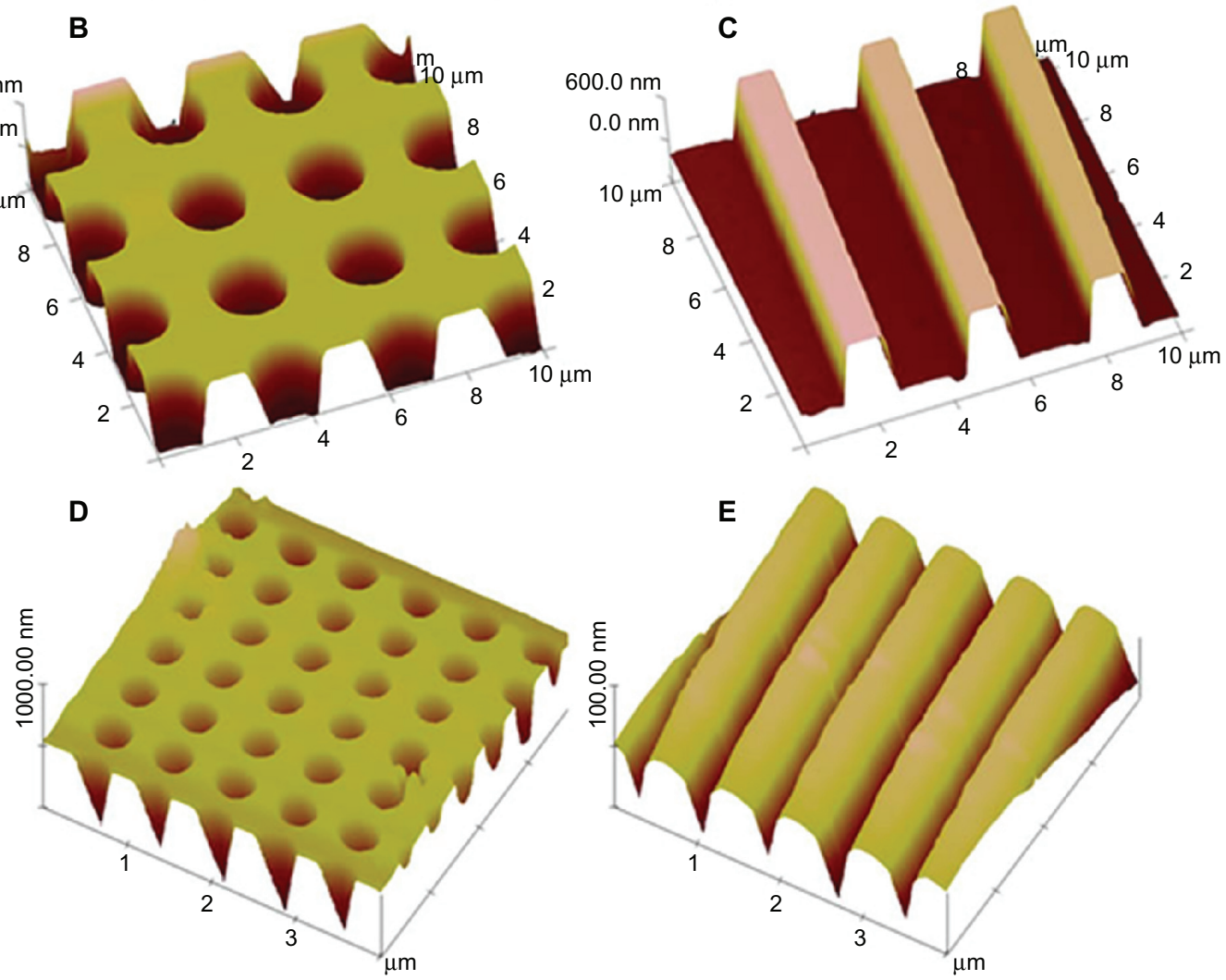

Figure I A Four-quadrant grid competition system. The grid competition system consists of four competition schemes, where each scheme is a competition between two topographies. The four-quadrant system incorporates homogeneous arrays of $\mathbf{B}$ (top left of $\mathbf{A}$ ) holes with a $2 \mu \mathrm{m}$ diameter with horizontal and vertical spacings of $\mathrm{I} \mu \mathrm{m}$ ( $3 \mu \mathrm{m}$ pitch), C (top right of A) lines of $2 \mu \mathrm{m}$ width with a spacing of I $\mu \mathrm{m}(3 \mu \mathrm{m}$ pitch), D (bottom left of A) holes with a $300 \mathrm{~nm}$ diameter with horizontal and vertical spacings of I $\mu \mathrm{m}(1.3 \mu \mathrm{m}$ pitch), and $\mathbf{E}$ (bottom right of $\mathbf{A}$ ) lines of $300 \mathrm{~nm}$ width with a spacing of I $\mu \mathrm{m}(1.3 \mu \mathrm{m}$ pitch). Structures all consisted of a depth of between $400-500 \mathrm{~nm}$. The unpatterned regions between each scheme are $20 \mu \mathrm{m}$ in width and are the areas where cells were positioned in the competition experiments.

a greater number of vertices; thus, defining an edge with a seemingly continuous curvature, like a simple circle, can require the specification of tens to hundreds of vertices. A circular structure (eg, a hole) created with an insufficient number of vertices would appear as a regular polygon with distinct edges instead of a circle with a continuously curved edge. Unfortunately, increasing the number of vertices defining a feature's boundaries involves a disproportionate increase in writing time. Accordingly, writing accurate submicroscale (and nanoscale) structures in terms of a closed polygon (area), regardless of boundary curvature (although curved objects take longer), typically takes a significant amount of time and depends both on the density of the structures in the array (structures/area) and the overall area to be patterned.

To reduce writing times significantly, the $300 \mathrm{~nm}$ holes and lines were patterned by single-pixel dot and line exposures in place of conventional area exposures. Individual pixels were essentially points (tiny areas) defined by the 
focused spot of the electron beam (usually a few nanometers in diameter). When single pixels were exposed, interactions between the electron beam and the electronically sensitive resist were allowed to spread symmetrically outward in a radial fashion to form a circle, the diameter of which depended on the fluence of the electron beam and the time at which each pixel was exposed (dwell time). Longer dwell times resulted in circles of larger diameters. Circles were formed by discrete single-pixel exposures using the beam shutter (blanker) while lines were formed by rastering the beam to form a continuous line of single pixels. For the $300 \mathrm{~nm}$ holes, dot fluence was set to $0.7 \mu \mathrm{C}$. For the $300 \mathrm{~nm}$ lines, line fluence was set to $2000 \mu \mathrm{C} / \mathrm{cm}$. Due to the larger dimensions of the $2 \mu \mathrm{m}$ structures, pattern density was small enough that the structures could be written as polygons with an area fluence of $100 \mu \mathrm{C} / \mathrm{cm}^{2}$; circular holes were drawn as regular polygons have 64 vertices. One thing to note is that the diameter of circles produced by irradiating single pixels expands slightly after the completion of the exposure because residual chemical reactions persist for short durations upon the blanking of the electron beam. Line widths from line exposures also expand slightly for the same reasons.

Arrays of simple objects, like holes or lines, can be written rather easily by implementing a strategically premeditated spatial arrangement of points or lines and appropriately setting beam parameters (ie, beam fluence and dwell time), which can be altered from pixel-to-pixel or line-to-line, over the area to be patterned. Arrays of more complex objects can be drawn by single-pixel writes as well, by creating the individual objects making up the array from a local compilation of dots and lines and copying the area to be patterned in the horizontal and vertical directions ( $x$ and $y$ directions in a Cartesian coordinate system). Many modern EBL tools include computer-aided drafting tools that make it possible to set up exposures graphically while permitting easy access to beam parameters. Dot and line exposures are quite useful for more hastily patterning arrays of submicroscale features on surfaces over large areas, which is often required of surfaces serving as substrates for biologic cells. Such was the case for our four-quadrant grid system; moreover, dot and line exposure could also be quite convenient in the fabrication of photonic crystal devices for optobiologic applications.

\section{Chemical pretreatment of quartz substrates}

Square wells of $1.5 \mathrm{~cm}^{2}$ inner area (the walls of the wells had a lateral thickness of several millimeters) were molded in polydimethylsiloxane (Slygard 184, Dow Corning,
Midland, MI). The wells were placed on each patterned quartz substrate, and sterilized by exposure to ultraviolet radiation for two hours. The polydimethylsiloxane rings were used to confine liquids on the substrates, which allowed us to conserve our liquid media, including the cell culture media containing the cell suspensions. Sterilized substrates were incubated in $0.1 \mathrm{mg} / \mathrm{mL}$ poly-D-lysine (Sigma-Aldrich Corporation, St Louis, MO) overnight and subsequently washed twice with sterile double-deionized water. Hydrated samples were dried in a sterile laminar flow bench and stored at $4^{\circ} \mathrm{C}$ until used in cell culture experiments.

\section{Isolation of rat hippocampal cells}

E18 rat embryonic hippocampal neurons were isolated from commercial rat hippocampal tissue (BrainBits, Springfield, IL) according to the manufacturer's protocol. The hippocampus was incubated in $4 \mathrm{mg} / \mathrm{mL}$ papain solution (Worthington, Lakewood, NJ) in Hibernate E medium (BrainBits) at $30^{\circ} \mathrm{C}$ for 20 minutes. A fire-polished Pasteur pipette was used to triturate the hippocampal tissue, followed by centrifugation ( $200 \mathrm{~g}$ for one minute). A cell pellet was suspended in $1 \mathrm{~mL}$ of warm culture medium containing Neurobasal medium (Invitrogen, Gaithersburg, MD), 2\% B-27 supplement (Invitrogen), $0.5 \mathrm{mM}$ L-glutamine (Fisher Scientific, Pittsburgh, PA), $0.025 \mathrm{mM}$ glutamic acid (Sigma-Aldrich), and $1 \%$ antibiotic-antimycotic solution (Sigma-Aldrich).

\section{Cell micropositioning}

Micropositioning techniques were employed to place cells in precise locations on the quartz substrates. Some hippocampal neurons were randomly seeded on the quartz substrates. Individual cells were repositioned in unpatterned gaps between the topographies (between the quadrants) in the four-grid competition system or at the borders of the topographies juxtaposing smooth surface using micropipettes and a specialized micropositioning system (see following subsections).

Tapered micropipettes were formed by pulling glass capillaries (single-barrel standard borosilicate glass tubing $1 \mathrm{~mm}$ outer diameter, $0.58 \mathrm{~mm}$ inner diameter, World Precision Instruments, Sarasota, FL) with a vertical pull type puller (PC-10, Narishige International, East Meadow, NY). The pulled micropipettes were connected to a pneumatic microinjector (IM-9C, Narishige International) and tightened to an XYZ movable micromanipulator (MN-151, Narishige International). This setup was mounted on a reflectance upright microscope (BX51WI, Olympus, Center Valley, PA) inside a horizontal laminar airflow workstation to guarantee sterility in the procedure. 
A patterned quartz substrate was placed inside a polydimethylsiloxane ring, which was placed in the center of a sterile Petri dish of $10 \mathrm{~cm}$ diameter. The size and thickness of the square ring (as opposed to circular) was small enough so that space was left between the outside wall of the square ring and the circular wall of the Petri dish. Triturated neurons in culture medium $\left(2 \times 10^{4}\right.$ cells $\left./ \mathrm{mL}\right)$ were added in the space outside the ring and allowed to settle for five minutes. Single neurons were identified on the Petri dish (outside the polydimethylsiloxane ring), aspirated with the micropipette by creating suction with the injector, moved with the micromanipulator, and repositioned inside the rings in desired locations on the quartz substrate by releasing from the pipette. After micropositioning, Petri dishes were incubated at $37^{\circ} \mathrm{C}$ and $5 \% \mathrm{CO}_{2}$ for 24 hours.

\section{Competition assays}

Topography versus smooth surface competition assays

To determine whether topography could guide axon growth relative to unpatterned smooth (bare) surface, individual embryonic hippocampal neurons were positioned at the outer fringes of the topographies (Figure 2A). Neurons were micropositioned at distances of approximately $30 \mu \mathrm{m}$ from the boundaries of the topographies. Multiple neurons were positioned around each topography before each competition experiment to facilitate a more rapid collection of data. After 24 hours in culture, the neurons were analyzed using
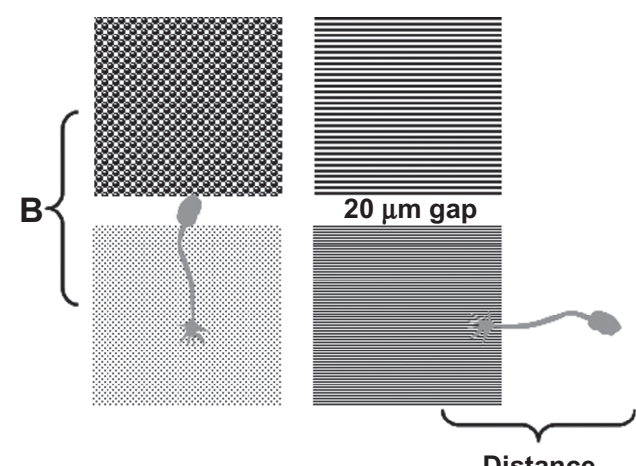

A

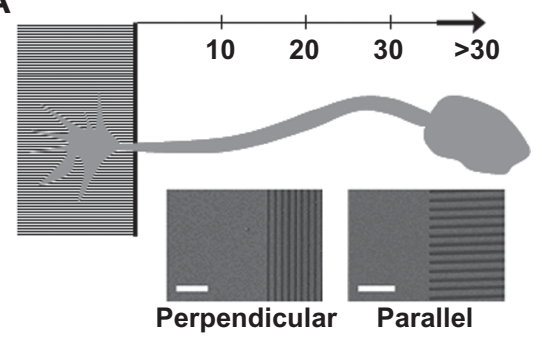

Figure 2 Cell positioning in competition experiments between (A) topography and unpatterned surface and (B) differing topographies. The scanning electron microscopy images in $\mathbf{A}$ denote the parallel and perpendicular boundary types for the lines. Notes: Scale bars $=5 \mu \mathrm{m}$. Distance in $\mu \mathrm{m}$. conventional optical and fluorescence microscopy. Statistical analyses using one-way analysis of variance (ANOVA), ${ }^{15}$ one- and two-sample binomial $t$-tests, ${ }^{16,17}$ and the Chi-squared $\left(\chi^{2}\right)$ test $^{18}$ was employed to determine whether results were significant.

Because it took the neurons some time to anchor themselves on the quartz substrates, the cells moved slightly from their initial position ( $30 \mu \mathrm{m}$ from the topography), most likely due to convection by the culture medium. We positioned the neurons at a distance of $30 \mu \mathrm{m}$ because we found that the average axon length of polarized hippocampal neurons on smooth quartz was approximately $30 \mu \mathrm{m}$ after 24 hours in culture. ${ }^{14}$ When a neuron extended and established its axon onto a certain topography, the topography was considered to be preferred over the smooth surface. Polarized cells were only analyzed when their axons were elongated enough to make contact with the neighboring topography. When an axon was sufficiently long, but did not touch topography, bare surface was regarded as the preferential substrate. Distance between the center of the cell body and the edge of the pattern was measured and reported as the "cellular distance". A distance of zero means that the center of the cell body of a neuron coincided with the pattern boundary.

Line patterns were divided into two types, perpendicular and parallel, because growing neurons and axons faced the grooves in two different ways (Figure 2A). The perpendicular boundary represented lines in which the boundary between the unpatterned and patterned region was created by a single line. The parallel boundary represented the other case where the boundary between the unpatterned and patterned region consisted of the ends of several lines. In total, four competition assays were conducted to compare preference for lines relative to smooth surface, ie, lines having the parallel and perpendicular boundary types for each size scale.

We performed two investigations involving the competitions between topography and smooth surface. First, we investigated axon formation and extension in neurons based on distance, as defined above, irrespective of feature shape, size, and boundary type. We compared cell preference in the ranges 0-10, 10-20, and $20-30 \mu \mathrm{m}$, with the preference $>30 \mu \mathrm{m}$. Preference data was consolidated for distances $<30 \mu \mathrm{m}$ and compared with the data for distances $>30 \mu \mathrm{m}$. Second, we compared preference based on the shape, size, and boundary type of topography for cells at distances $<30 \mu \mathrm{m}$.

\section{Topography versus topography competition assays}

After performing competitions between topography and smooth surface, competition studies were performed 
between topographies to evaluate axon preference further based on feature size and shape (Figure 2B). Individual hippocampal neurons were micropositioned in the $20 \mu \mathrm{m}$ unpatterned regions between neighboring topographies and cultured for 24 hours. For each micropositioned neuron, the topography drawing the axon to it was counted as the preferred topography (ie, winner of the competition).

\section{Immunofluorescence}

Embryonic hippocampal neurons cultured on the substrates were fixed with $4 \%$ paraformaldehyde (Sigma-Aldrich) and $4 \%$ sucrose (Sigma-Aldrich) in phosphate-buffered saline ( $\mathrm{pH}$ 7.2) for 20 minutes at room temperature. Fixed samples were permeabilized with $0.1 \%$ Triton X-100 (Fluka, St Louis, MO) and 3\% goat serum (Sigma-Aldrich) in phosphate-buffered saline buffer for 20 minutes, washed twice with phosphatebuffered saline, and treated with a blocking solution of 3\% goat serum in phosphate-buffered saline for one hour at $37^{\circ} \mathrm{C}$. Tau-1, a microtubule protein expressed in axons, was labeled as an axonal marker. Mouse tau- 1 antibody (Chemicon, Temecula, CA) was diluted to 1:200 in blocking solution, and added to the culture samples. After overnight incubation at $4^{\circ} \mathrm{C}$, the samples were washed with phosphate-buffered saline two times, treated with a secondary antibody solution of Alexa 488-labeled goat antirat IgG (Invitrogen, 1:200 dilution in blocking solution) at $4^{\circ} \mathrm{C}$ for five hours, and rinsed in phosphate-buffered saline for five minutes two times. Samples were stored at $4^{\circ} \mathrm{C}$ while awaiting further analysis.

\section{Distance measurements based}

on immunofluorescence

Fluorescence images of cells and axons were acquired using a fluorescence microscope (IX-70, Olympus). The images were captured using a color CCD camera (Optronics MagnaFire, Goleta, CA). Cell images were analyzed using Image J software (available from the National Institutes of Health website). Distance was routinely measured as the linear distance between the center of the cell body and the edge of the topography associated with the measurement. When several neurites branched from a single nerve cell, the longest neurite was used in the measurement. A neuron was considered to be polarized only when the axon was approximately two times longer than the characteristic diameter of the cell body. The stages of neuron development are documented in Dotti et a ${ }^{15}$ and Goslin and Banker. ${ }^{16}$

\section{Topography and cells under scanning electron and atomic force microscopy}

Fixed hippocampal neurons were dehydrated by treating with ethanol in water at successively increasing concentrations; cells were treated at concentrations $(\mathrm{v} / \mathrm{v})$ of $30 \%$ for 45 minutes, $50 \%$ for 30 minutes, and $70 \%, 85 \%, 90 \%, 95 \%$, and absolute ethanol (100\%, Pharmco, Brookfield, CT) for 10 minutes each. Water was completely removed by adding hexamethyldisilazane (Sigma-Aldrich) and drying in air at ambient conditions. The dried samples were coated with a thin $10 \mathrm{~nm}$ layer of platinum/palladium by sputter coating (208HR, Cressington Scientific Instruments, Watford, UK). Scanning electron microscope images were acquired with a Zeiss SUPRA 40 VP Scanning Electron Microscope (Carl Zeiss, Peabody, MA). Atomic force microscopy images were taken to ensure precise dimensions of topography. Atomic force microscopy images were acquired with a Dimension 3100 with Nanoscope IV controller (Digital Instruments and Veeco Metrology Group, Santa Barbara, CA) using a silicon tip in tapping-mode (Tap300, Budget Sensors, Sophia, Bulgaria).

\section{Statistical analysis of experimental data}

Cell count data was analyzed using a combination of balanced one-way analysis of variance (ANOVA),${ }^{17}$ one- and two-sample binomial $t$-tests, ${ }^{18,19}$ and Chi-squared $\left(\chi^{2}\right)$ tests. ${ }^{20}$ A $50 \%$ probability distribution was assumed in calculating test statistics in the one-sample binomial and $\chi^{2}$ tests. One-sample binomial $t$-tests were performed in lieu of $\chi^{2}$ tests when sample sizes were small. In all other cases where data were compared with a $50 \%$ probability, only $\chi^{2}$ tests were performed. Both the two-sample binomial $t$-tests and one-way ANOVAs were performed together to determine whether individual averages were statistically different; in most cases, results for the tests are presented together. $P$ values were interpolated from standard $t$-tests and $\chi^{2}$ distribution tables using the observed test statistics. Averages were deemed statistically different if significant to greater than a $95 \%$ confidence level according to the $P$ value $(P<0.05)$. Significance to greater than a $90 \%$ confidence level is indicated (eg, $P$ values $\approx 0.5-0.1$ ), but not deemed to be statistically significant. In some cases where samples were not statistically significant, or in cases where statistical tests were deemed inappropriate, observations and/or trends were noted. To note, we do not preclude the existence of moderate Type II error in statistical calculations in which significance was not determined; thus, post hoc statistical power analysis is considered for select insignificant statistical comparisons.

\section{Results}

\section{Competitions between topography} and smooth surface

Experimental protocols describing the competitions between topography and smooth surface can be found in the Materials 


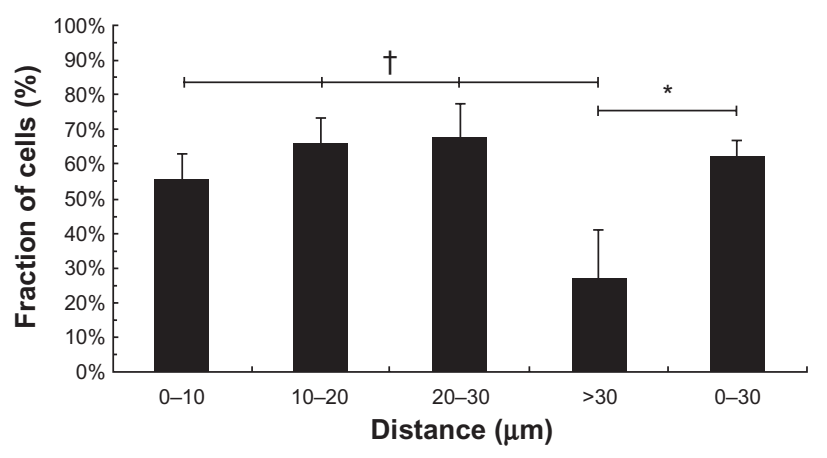

Figure 3 Fraction of cells (\%) choosing topography over smooth surface based on the distance measured from the center of the cell body to the topography boundary. Error bars $=$ standard error of the mean; distance units $=\mu \mathrm{m}$. One-way analysis of variance (ANOVA) and two-sample binomial $t$-tests were used to compare the data for statistical significance. $\chi^{2}$ tests were conducted to determine whether preference was statistically different from a $50 \%$ probability choice.

Notes: $P$ values are indicated for each competition. $* 0-30$ versus $>30, P<0.05$ (ANOVA and binomial); $\chi^{2}$ tests: $0-30, P<0.05,>30$, not significant. 'Preferences in the $0-10,10-20$, and 20-30 ranges were not significant relative to one another, but each range $<30 \mu \mathrm{m}$, except $0-10$, was statistically different than $>30$ to greater than a 95\% confidence ( $10-20$ and $20-30$ versus $>30, P<0.05,0-10$ versus $>30$, $P \leq 0.1$, ANOVA and binomial). Sample sizes ( $n=$ number of cells): $0-10, n=38$; $10-20, \mathrm{n}=35 ; 20-30, \mathrm{n}=25 ; 0-30, \mathrm{n}=98 ;>30, \mathrm{n}=1 \mathrm{I}$.

and methods section (also see Figure 2A). Figure 3 and Figure 4 summarize the results of the competitions between the topographies and smooth surface. Figure $5 \mathrm{~A}-\mathrm{F}$ show representative images of neurons positioned on the outer borders of the topographies in the grid.

\section{Preference based on distance}

We determined the overall axonal preference for topography relative to smooth surface based on distance regardless of

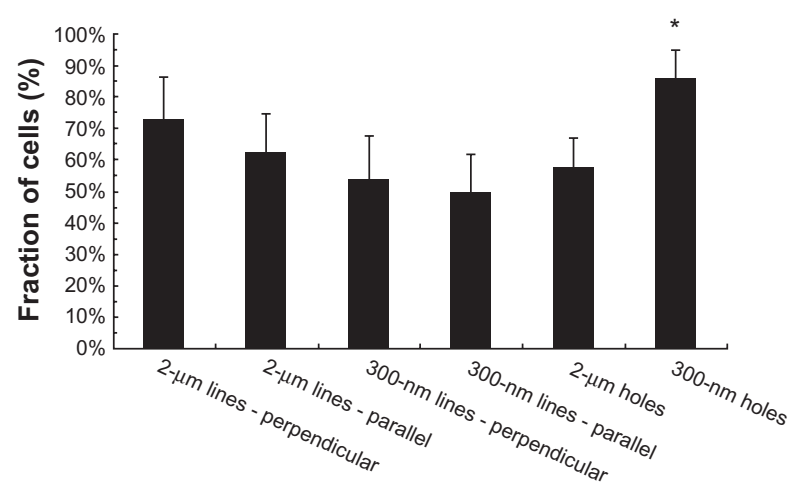

Figure 4 Fraction of cells choosing topography (\%) based on feature size, shape, and boundary type (perpendicular and parallel for line topographies) for distances less than $30 \mu \mathrm{m}$. Distance was measured as the length between the center of the cell body to the topography boundary. Scale bars = standard error of the mean. Two-sample binomial $t$-tests were used to compare the data for statistical significance. Preference for the $300 \mathrm{~nm}$ holes was found to be statistically significant relative to preference on the other topographies except for the $2 \mu \mathrm{m}$ lines of both boundary types (parallel and perpendicular); moreover, preferences for all the other topographies were not statistically different relative to one another.

Notes: $* 300 \mathrm{~nm}$ holes versus all other topographies except $2 \mu \mathrm{m}$ lines (both boundary types), $P<0.05$ (binomial). Sample sizes ( $n=$ number of cells): $2 \mu \mathrm{m}$ lines (perpendicular), $\mathrm{n}=1 \mathrm{I} ; 2 \mu \mathrm{m}$ lines (parallel), $\mathrm{n}=16 ; 300 \mathrm{~nm}$ lines (perpendicular), $\mathrm{n}=13 ; 300 \mathrm{~nm}$ lines (parallel), $\mathrm{n}=18 ; 2 \mu \mathrm{m}$ holes, $\mathrm{n}=26 ; 300 \mathrm{~nm}$ holes, $\mathrm{n}=14$. feature shape, size, and boundary orientation (Figure 2A, Figure 3). For each cell, distance was measured from the center of the cell body to the boundary of the particular topography in the competition (for information about the protocol, see Materials and methods); 55.3\%, 65.7\%, $68.0 \%$, and $27.3 \%$ of neurons extended their axons towards topography for distances between 0-10, 10-20, 20-30, and $>30 \mu \mathrm{m}$, respectively. The consolidated weighted average of cells choosing topography for distances $<30$ $\mu \mathrm{m}$ was $62.2 \%(\mathrm{n}=98)$, which differed statistically from a $50 \%$ probability choice; cell preference for cells positioned at $>30 \mu \mathrm{m}(27.3 \%, \mathrm{n}=11)$ did not differ from a $50 \%$ probability $\left(P<0.05, \chi^{2}\right.$ tests $)$. Cell preference for topography for distances $<30 \mu \mathrm{m}$ was statistically significant relative to cell preference for topography at distances $>30 \mu \mathrm{m}(P<0.05$, binomial and ANOVA). ANOVA and binomial tests were also used to determine whether preferences for topography in the specific distance ranges of 0-10 (55.3\%), 10-20 (65.7\%), and 20-30 (68.0\%) $\mu \mathrm{m}$ were significantly different from the $27.3 \%$ preference for $>30$ $\mu \mathrm{m}$ distances. Cells within 10-20 and 20-30 $\mu \mathrm{m}$ exhibited a preference that was statistically different than those $>30 \mu \mathrm{m}$ $(P<0.05)$; however, cell preference within $0-10 \mu \mathrm{m}$ did not differ significantly from $>30 \mu \mathrm{m}(P \leq 0.1)$. One would assume that because the preference for topography was significant in the ranges of $10-20 \mu \mathrm{m}$ and $20-30 \mu \mathrm{m}$ relative to $>30 \mu \mathrm{m}$, preference between $0-10 \mu \mathrm{m}$ would also have been significant relative to $>30 \mu \mathrm{m}$; thus, we suspect that this discrepancy is due to inherent Type II error resulting from an insufficient sample size in the $0-10 \mu \mathrm{m}$ range. Finally, ANOVA was used to determine whether preferences in the ranges of $0-10,10-20$, and $20-30 \mu \mathrm{m}$ were statistically different from one another; however, we found that the differences were not significant.

\section{Preference based on feature size, shape, and boundary-type for close distances}

Based on the $30 \mu \mathrm{m}$ limiting distance, we analyzed preference based on feature shape, size, and boundary type (parallel and perpendicular for the lines) for neurons that were positioned at distances $<30 \mu \mathrm{m}$. Figure 4 tabulates the results of the competitions. The portion of neurons extending axons to topography was $57.5 \%$ and $85.7 \%$, for the $2 \mu \mathrm{m}$ holes and the $300 \mathrm{~nm}$ holes, respectively. For the $300 \mathrm{~nm}$ lines, $53.8 \%$ and $50.0 \%$ of neurons selected the $300 \mathrm{~nm}$ lines of perpendicular and parallel boundaries for their axonal growth over smooth surface, respectively; $72.7 \%$ and $62.5 \%$ of neurons extended axons to the $2 \mu \mathrm{m}$ lines of 

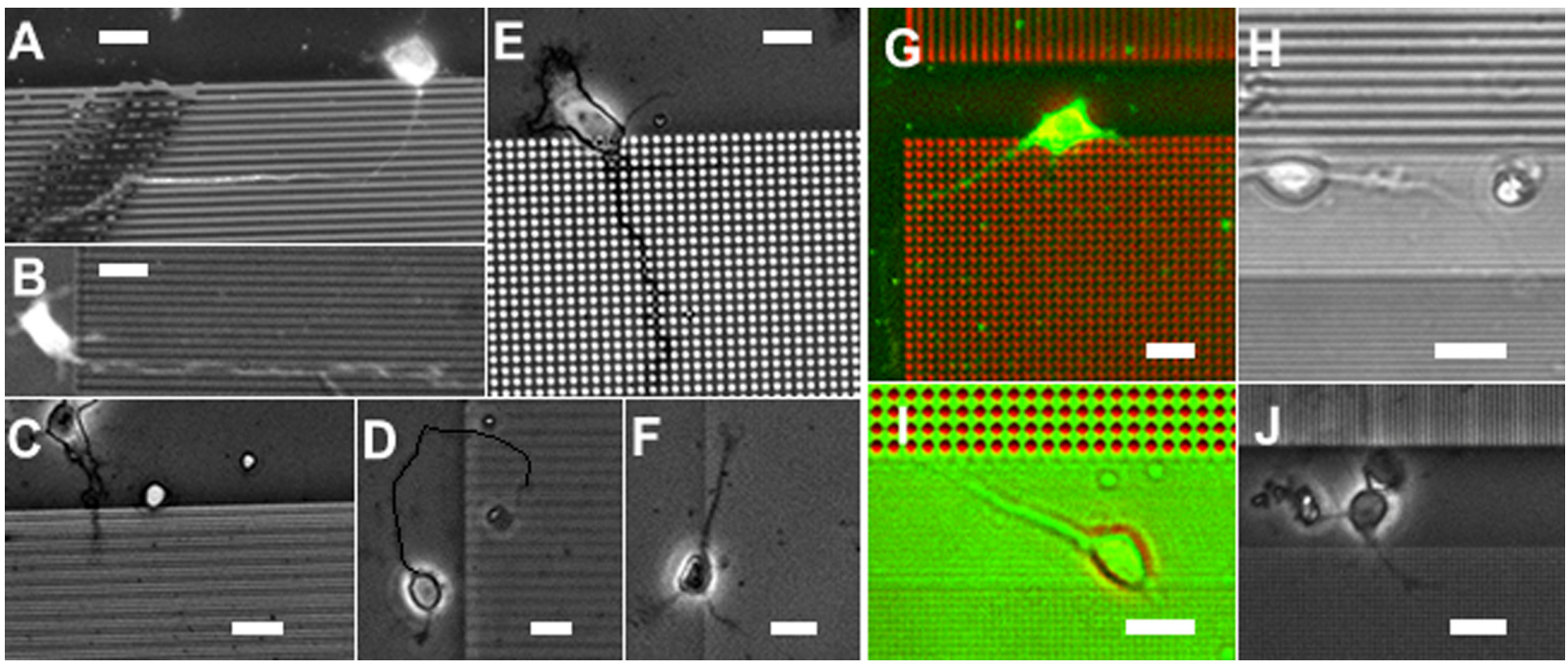

Figure 5 Optical images (in some cases labeled with tau-I and 4,6-diamidino-2-phenylindole) of neurons establishing their axons on the topographies. Images A-E show neurons micropositioned on the outer borders of the topographies and illustrate competitions between bare surface and the (A) $2 \mu \mathrm{m}$ lines (perpendicular), (B) $2 \mu \mathrm{m}$ lines (parallel), (C) $300 \mathrm{~nm}$ lines (perpendicular), (D) $300 \mathrm{~nm}$ lines (parallel), (E) $2 \mu \mathrm{m}$ holes, and (F) $300 \mathrm{~nm}$ holes. Lines of perpendicular and parallel borders are shown in (A, C and (B, D) respectively. Images (G-J) show neurons micropositioned in the unpatterned spaces ( $20 \mu \mathrm{m}$ wide) between the topographies in the four-quadrant grid. The images illustrate competitions between (G) $2 \mu \mathrm{m}$ lines and $2 \mu \mathrm{m}$ holes, (H) $2 \mu \mathrm{m}$ lines and $300 \mathrm{~nm}$ lines, (I) $2 \mu \mathrm{m}$ holes and $300 \mathrm{~nm}$ holes, and (J) $300 \mathrm{~nm}$ lines and $300 \mathrm{~nm}$ holes.

Notes: Scale bars $=10 \mu \mathrm{m}$. (Image D has a curve superimposed on the axon to improve visibility; color has been added to images G, I to improve visibility).

perpendicular and parallel boundaries, respectively. Statistical tests showed that only the preference for the $300 \mathrm{~nm}$ holes $(85.7 \%)$ was statistically significant relative to the $2 \mu \mathrm{m}$ holes and $300 \mathrm{~nm}$ lines of boundary-types $(P<0.05$, binomial tests); preference for the $300 \mathrm{~nm}$ holes was not significant relative to the $2 \mu \mathrm{m}$ lines (of both boundary types). Preferences for all the other topographies and boundary types did not differ statistically relative to one another.

Based on the competition experiments comparing topography with smooth surface, it appeared that distance affected axon preference, and that the $300 \mathrm{~nm}$ holes served as the strongest cue of any of the topographies.

\section{Competitions between topographies}

Experimental protocols describing the competitions between the topographies can be found in the Materials and methods section (see Figure 2B). Figure 6 summarizes the results of the competitions between the topographies. Figure $5 \mathrm{G}-\mathrm{J}$ shows representative images of neurons positioned in the gaps between neighboring topographies in the topography grid.

\section{Preference based on feature size}

To investigate preference based on feature size, we compared the $2 \mu \mathrm{m}$ lines with the $300 \mathrm{~nm}$ lines and the $2 \mu \mathrm{m}$ holes with the $300 \mathrm{~nm}$ holes, and found that $100 \%$ of neurons extended their axons onto the $300 \mathrm{~nm}$ lines rather than the $2 \mu \mathrm{m}$ lines, while $75 \%$ of neurons extended their axons onto the $2 \mu \mathrm{m}$ holes rather than the $300 \mathrm{~nm}$ holes. One-way binomial tests were used to determine preference relative to a $50 \%$ probability. Preference for the $300 \mathrm{~nm}$ lines (over the $2 \mu \mathrm{m}$ lines) was statistically significant to slightly greater than a $95 \%$ confidence $(P<0.05$, binomial). Due to a relatively small sample size in the comparison of the holes, power analysis revealed that Type II error could have precluded us from obtaining a significant result. The data for the lines is consistent with results published by Gomez et al ${ }^{13}$ who found that axons preferred smaller lines of $1 \mu \mathrm{m}$ width over larger lines of $2 \mu \mathrm{m}$ width.

\section{Preference based on feature shape}

Competitions were studied between holes and lines of similar dimension to determine preference based on feature

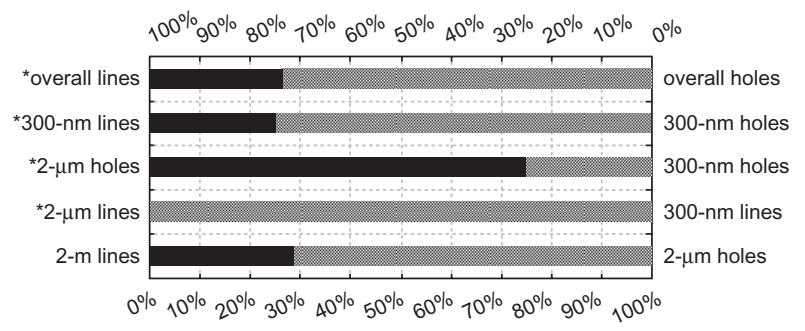

Figure 6 Results of the competitions between topographies. The black and dotted bars represent the percentage of cells choosing the topographies on the left and right vertical axes, respectively. One-sample binomial $t$-tests were conducted to determine whether preference was statistically different than a $50 \%$ probability distribution. Notes: $* P \approx 0.05$. Sample sizes $(n=$ number of cells): $2 \mu \mathrm{m}$ lines versus $2 \mu \mathrm{m}$ holes, $\mathrm{n}=7 ; 2 \mu \mathrm{m}$ lines versus $300 \mathrm{~nm}$ lines, $\mathrm{n}=6$; $2 \mu \mathrm{m}$ holes versus $300 \mathrm{~nm}$ holes, $\mathrm{n}=4$; $300 \mathrm{~nm}$ lines versus $300 \mathrm{~nm}$ holes, $\mathrm{n}=8$; overall lines versus overall holes, $\mathrm{n}=15$. 
shape $(2 \mu \mathrm{m}$ holes versus $2 \mu \mathrm{m}$ lines and $300 \mathrm{~nm}$ holes versus $300 \mathrm{~nm}$ lines). In these cases, lines had the parallel boundaries; $71.4 \%$ of neurons extended their axons towards the $2 \mu \mathrm{m}$ holes over the $2 \mu \mathrm{m}$ lines, and $75 \%$ of neurons chose the $300 \mathrm{~nm}$ holes over the $300 \mathrm{~nm}$ lines. One-way binomial tests were used to determine preference relative to a $50 \%$ probability. Neither of the results was found to be statistically significant; however, based on our relatively small sample sizes, Type II error could have generated some false negative outcomes. In compiling the data for the assays comparing morphology, ie, overall lines versus holes, $73.3 \%$ of cells chose holes over lines to about a $95 \%$ confidence $(P=0.06$, binomial $)$, which indicates that neurons may have preferentially established their axons on holes relative to lines.

\section{Discussion}

In the body, neurons are naturally encompassed by a network of physical (topographic) structures and boundary conditions that signal/cue the maturation of neural processes in a highly aligned fashion. ${ }^{21}$ The maturation of neural processes involves the formation, growth, and orientation of an axon from a single neurite. Accordingly, harnessing the power of these naturally occurring phenomena by engineering a well-defined network of physical cues onto extracellular matrix-mimicking biomaterial scaffolds may effectively enhance our capability to modulate specific nerve cell responses. ${ }^{22,23}$ The question then arises as to which kind of physical cues are most useful in provoking the kind of responses that we seek.

The competitions between topography and smooth surface demonstrated that topography influences developing immature neurons relative to a flat surface, assuming the same choice of material. Moreover, neurons growing in close proximity to the topographies appeared to enable an immature neurite to become an axon. At a distance of $>30 \mu \mathrm{m}$, topography had little effect on axon selection. At distances $<30 \mu \mathrm{m}$, topography appeared to induce a neuronal response evident by an increase in axon sequestration. Thus, a distance of close to $30 \mu \mathrm{m}$ appears to serve as a limit at which topographic features become too distant for growing neurites and axons to sense after only 24 hours of culture.

The idea that a cue has to be within a reasonable distance from a cell to impose its presence on the cell seems rather intuitive. One could presume that an immature neuron is able to sense topography from a distance, albeit a relatively small distance $(30 \mu \mathrm{m})$, so that physical cues need not be in direct contact with the neuron to provoke them.
Moreover, based on these results, we speculate as to how physical topography biochemically affects neurons, ie, how interactions between a neuron and a physical feature tune the intracellular mechanisms relevant to axon development and the way in which a neuron interacts with and/or responds to its environment.

Microscale and submicroscale topography introduce physical discontinuities in surface area that apply tractive mechanical stresses to attached cells. Stresses imposed on anchored neurons set off a chain of intracellular events that ultimately leads to modified responses. The exact intracellular mechanisms by which surface texture affects neuronal behavior are not clearly understood to this point, although one would assume that, indeed, topography induces cytoskeletal reorganization, changes in cell shape, and changes in the distribution of focal adhesions, which ultimately do trigger altered responses. Lee et $\mathrm{al}^{24}$ proposed that topography initiates alterations in focal adhesions by causing distortions in the cytoskeleton, which trigger intracellular mechanisms that control axon initiation. It has also been reported that external mechanical forces applied to the integrinextracellular matrix adhesions of anchorage-dependent cells strengthens the integrin receptors as they mature into focal complexes and adhesions. Upon the formation of focal complexes and, sometimes, larger focal adhesions, the cell is able to apply forces on its surroundings, which allow the cell to strengthen its grip on its extracellular matrix or to migrate. ${ }^{25}$ The ability of neurons to generate force is related to their actin-based dynamic mode of motility, where actin meshworks assemble at the leading edge of their lamellipodium, translocate backwards from the leading edge, and later depolymerize for recycle. ${ }^{26}$ Thus, we hypothesize that the application of externally applied stresses, eg, due to the introduction of physical topography, modulate the organization of actin filaments in the growth cone of single neurites and the growth and assembly of intermediate filaments and microtubules in the axon. This remodeling alters the degree to which neurons polarize, the axonal growth rate, and axonal orientation. It has also been suggested that mechanical stresses abet altered cellular responses by transducing changes in gene expression. ${ }^{6,8}$

Although it is evident that topography affects the polarization of immature neurons, and that the precise cascade of intracellular events leading to this rectified behavior is rather esoteric, we ask the question as to whether the size and shape of topography affect axon formation and growth. In our experiments, we originally conjectured that neurons may 
sense the submicroscale and microscale topographies differently, at a fundamental level, due to the order-of-magnitude difference in size scale. Because of their relatively small size, submicroscale pitted features ( $300 \mathrm{~nm}$ ), eg, holes and grooves, are largely inaccessible by cellular structures, thus, rendering the surfaces heterogeneous in terms of material properties where the trough regions (grooves) represent pockets of fluid (culture medium). The pockets of fluid, in combination with the solid surface, collectively would change the properties of the surface recognized by cells having sizes of larger scale. On the other hand, because cells are able to access the grooves of larger microscale features $(2 \mu \mathrm{m})$, the microscale features may serve to introduce physical discontinuities into surface area, but not change material properties from the perspective of individual cells. ${ }^{27-30}$ This fundamental difference in the way a neuron perceives topography, based on size scale, would leave one to believe that order-of-magnitude changes in feature size activate alternate intracellular pathways, which would cause the cells to behave differently.

It has been proposed that cell adherence depends on the proportion of ridge area on patterned surfaces, which depends on feature size and density. Teixeira ${ }^{9}$ found that corneal epithelial cells on different patterns (ie, smooth surface, microscale, and nanoscale lines) displayed different focal adhesion numbers and sizes. As ridge-widths decreased, focal adhesion sizes decreased, commensurately altering the adhesive properties of the topographies. Sapelkin et $\mathrm{al}^{31}$ observed that immortalized rat hippocampal neurons preferentially adhered to porous silicon than crystalline silicon. Previous studies have reported that smaller features have a greater impact on polarization.

Gomez et $\mathrm{al}^{32}$ found that polydimethylsiloxane microchannels of $1 \mu \mathrm{m}$ and $2 \mu \mathrm{m}$ width enhanced axon formation in rat embryonic hippocampal neurons compared with smooth polydimethylsiloxane after 20 hours in culture. Furthermore, the neurons preferred the smaller lines of $1 \mu \mathrm{m}$ width over the larger lines of $2 \mu \mathrm{m}$ width. It has been suggested that preference for smaller lines, of $1 \mu \mathrm{m}$ width or less, may be due to the fact that the particular size scale mimics the sizes characteristic of neurite fibers found in peripheral nerves in vivo. ${ }^{33}$ Lee at a ${ }^{24}$ cultured hippocampal neurons on various polymer poly(lactic-co-glycolic acid, PLGA) fibers having diameters of $400 \mathrm{~nm}$ to $2.2 \mu \mathrm{m}$ and found that a greater number of neurons polarized on smaller PLGA fibers, while differences in fiber orientation for similar fiber diameters had a negligible effect on polarization. In the competitions between smooth surface and topography, for distances $<30 \mu \mathrm{m}$, we found that the $300 \mathrm{~nm}$ holes elicited polarization to a greater degree than the other topographies (Figure 4). Also, in the competitions comparing topographies, we found that the $300 \mathrm{~nm}$ lines were a stronger physical cue than $2 \mu \mathrm{m}$ lines, which is consistent with the idea that smaller features have a greater impact on polarization (Figure 6).

As to how differences in feature shape affect cell behavior, like with the case of changes in feature size, different shapes may transduce a different intracellular response in immature neurons resulting in different behavior. Moreover, this refashioned intracellular response may be due to a difference in the spatial distribution of mechanical stresses inflicted on the neuron. In addition to changes in feature size, it could be conjectured that changes in feature size would also induce changes in cell behavior based on differences in spatial stress distributions. However, it seems logical that differences in feature shape would have a greater effect on stress distributions, especially in the case holes with lines, than changes in feature size, eg, $2 \mu \mathrm{m}$ to $300 \mathrm{~nm}$.

It is still unclear as to how a neuron decides which neurite to transform and elongate into an axon. Selection of a neurite to form into an axon is considered random; however, external cues, such as topography, may provide an inductive signal to a specific neurite near the topography coercing it to form into an axon and elongate. It has been reported that neurons exhibit feedback loops, which either inhibit the growth of a neurite (negative loop) or signal its transformation into an axon (positive loop). ${ }^{34}$ Due to the fact that topography provides a stimulative cue to neurons to form an axon, we speculate that the topography encourages the execution of the positive loop in a greater proportion of neurons. Lamoureux et $\mathrm{al}^{35}$ were able to coerce an individual immature neurite to transform into an axon by applying tension to their growth cones with a micropipette. In close agreement with what has been surmised in the literature, ${ }^{13}$ we speculate that topography may have a similar effect on neurons, although acting as a more passive stimulus than a micropipette. Moreover, we suspect that the particular neurite that gets stimulated depends upon the orientation of the soma and the spatial distribution of neurites on the immature neuron upon anchoring itself to its substrate.

\section{Conclusion}

Competition assays were performed to determine the ability of topography to entice axon formation based on feature size and shape. We designed a topography grid consisting of arrays of holes and lines of $2 \mu \mathrm{m}$ and $300 \mathrm{~nm}$ critical 
dimension. Single neurons were micropositioned in gaps between neighboring topographies or at the border of individual topographies juxtaposing unpatterned smooth surface, and axon preference was determined after a 24-hour culture. We found that neurons positioned in close proximity to topography $(<30 \mu \mathrm{m})$ appeared to recognize and respond to the topography, regardless of feature dimensions or shape; in addition, the $300 \mathrm{~nm}$ holes were found to be a stronger cue than the other topographies. In the competitions between the topographies, neurons extended their axons towards the $300 \mathrm{~nm}$ lines at a greater frequency than the $2 \mu \mathrm{m}$ lines, which is consistent with trends reported in the literature showing that smaller lines are more stimulative than larger lines. ${ }^{14}$ The competitions between lines and holes indicated that the neurons preferred holes over lines, provided that the different features have an equivalent critical dimension.

The results reported here suggest the efficacy of implementing physical cues of various shapes and sizes on nerve guidance conduits and other advanced biomaterial scaffolds. Furthermore, we believe that further investigations need to be conducted to assess more adequately the stimulative effects of topography on neuronal development and to understand the associated intracellular mechanisms which cause neurons to alter their responses.

\section{Acknowledgments}

The contribution of CES to this project was supported by the National Institutes of Health (NIH R01EB004429). Work was performed at the Center for Nano and Molecular Science and Technology (CNM), Microelectronics Research Center (MRC), a part of the National Nanofabrication Infrastructure Network supported by the National Science Foundation (NSF), and Texas Materials Institute (TMI) at the University of Texas at Austin.

\section{Disclosure}

The authors report no conflicts of interest in this work.

\section{References}

1. Mattson MP. Establishment and plasticity of neuronal polarity. J Neurosci Res. 1999;57:577-589.

2. Lazic SE, Barker RA. The future of cell-based transplantation therapies for neurodegenerative disorders. J Hematother Stem Cell Res. 2003; $12: 635-642$.

3. Schmidt CE, Leach JB. Neural tissue engineering: Strategies for repair and regeneration. Annu Rev Biomed Eng. 2003;5:293-247.

4. Guenard V, Kleitman N, Morrissey TK, Bunge RP, Aebischer P. Syngeneic schwann-cells derived form adult nerves seeded in semipermeable guidance channels enhance peripheral-nerve regeneration. J Neurosci. 1992;12:3310-3320.
5. Schmidt CE, Shastri VR, Vacanti JP, Langer R. Stimulation of neurite outgrowth using an electrically conducting polymer. Proc Natl Acad Sci U S A. 1997;94:8948-8953.

6. Curtis A, Wilkinson C. Topographical control of cells. Biomaterials. 1997; 18:1573-1583.

7. Dalby MJ, Riehle MO, Yarwood SJ, Wilkinson CDW, Curtis ASG. Nucleus alignment and cell signaling in fibroblasts: Response to a micro-grooved topography. Exp Cell Res. 2003;284:274-282.

8. Dalby MJ. Topographically induced direct cell mechanotransduction. Med Eng Phys. 2005;27:730-742.

9. Teixeira AI, Abrams GA, Bertics PJ, Murphy CJ, Nealey PF. Epithelial contact guidance on well-defined micro- and nanostructured substrates. J Cell Sci. 2003;116:1881-1892.

10. Teixeira AI, Abrams GA, Murphy CJ, Nealey PF. Cell behavior on lithographically defined nanostructured substrates. J Vac Sci Technol B. 2003;21:683-687.

11. Rajnicek AM, Britland S, McCaig CD. Contact guidance of CNS neurites on grooved quartz: Influence of groove dimensions, neuronal age and cell type. J Cell Sci. 1997;110:2905-2913.

12. Rajnicek AM, McCaig CD. Guidance of CNS growth cones by substratum grooves and ridges: Effects of inhibitors of the cytoskeleton, calcium channels and signal transduction pathways. J Cell Sci. 1997;110: 2915-2924.

13. Gomez N, Chen SC, Schmidt CE. Polarization of hippocampal neurons with competitive surface stimuli: Contact guidance cues are preferred over chemical ligands. J Royal Soc Interface. 2007;4:223-233.

14. Fozdar DY, Lee JY, Schmidt CE, Chen S. Neurons respond uniquely to topographies of various sizes and shapes. Biofabrication. 2010;2: 035005 .

15. Dotti CG, Sullivan CA, Banker GA. The establishment of polarity by hippocampal-neurons in culture. J Neurosci. 1988;8:1454-1468.

16. Goslin K, Banker G. Experimental-observations on the development of polarity by hippocampal-neurons in culture. J Cell Biol. 1989;108: 1507-1516.

17. Christensen R. One-way analysis of variance. In: Analysis of Variance, Design and Regression. London: Chapman and Hall; 1991.

18. Christensen R, One binomial sample. In: Analysis of Variance, Design and Regression. London: Chapman and Hall; 1991.

19. Christensen R. Two independent binomial samples. In: Analysis of Variance, Design and Regression. London: Chapman and Hall; 1996.

20. Christensen R. One multinomial sample. In: Analysis of Variance, Design and Regression. London: Chapman and Hall; 1996.

21. Brittis PA, Canning DR, Silver J. Chondroitin sulfate as a regulator of neuronal patterning in the retina. Science. 1992;255:733-736.

22. Liu CY, Westerlund U, Svensson M, et al. Artificial niches for human adult neural stem cells: Possibility for autologous transplantation therapy. J Hematother Stem Cell Res. 2003;12:689-699.

23. Stenger DA, Hickman JJ, Bateman KE, et al. Microlithographic determination of axonal/dendritic polarity in cultured hippocampal neurons, J Neurosci Methods. 1998;82:167-173.

24. Lee JY, Bashur CA, Gomez N, Goldstein AS, Schmidt CE. Enhanced polarization of embryonic hippocampal neurons on micron scale electrospun fibers. J Biomed Mater Res A. 2010;92 A:1398-1406.

25. Galbraith CG, Yamada KM, Sheetz MP. The relationship between force and focal complex development. J Cell Biol. 2002;159:695-705.

26. Cameron LA, Giardini PA, Soo FS, Theriot JA. Secrets of actin-based motility revealed by a bacterial pathogen. Nat Rev Mol Cell Biol. 2000;1: 110-119.

27. Cassie ABD, Baxter S. Wettability of porous surfaces. Trans Faraday Soc. 1944;40:546-551.

28. Kim P, Kim DH, Kim B, et al. Fabrication of nanostructures of polyethylene glycol for applications to protein adsorption and cell adhesion. Nanotechnology. 2005;16:2420-2426.

29. Adamson AW. The solid-liquid interface - contact angle. In: Physical Chemistry of Surfaces. New York, NY: John Wiley and Sons; 1982. 
30. Fozdar DY, Wu X, Patrick CW, Chen S. Micro-well texture printed into PEG hydrogels using the FILM nanomanufacturing process affects the behavior of preadipocytes. Biomed Microdevices. 2008;10: 839-849.

31. Sapelkin AV, Bayliss SC, Unal B, Charalambou A. Interaction of B50 rat hippocampal cells with stain-etched porous silicon. Biomaterials. 2006;27:842-846.

32. Gomez N, Lu Y, Chen SC, Schmidt C. Immobilized nerve growth factor and microtopography have distinct effects on polarization versus axon elongation in hippocampal cells in culture. Biomaterials. 2007;28: 271-284.
33. Nagata I, Kawana A, Nakatsuji N. Perpendicular contact guidance of CNS neuroblasts on artificial microstructures. Development. 1993;117: 401-408.

34. Arimura N, Kaibuchi K. Key regulators in neuronal polarity. Neuron. 2005;48:881-884.

35. Lamoureux P, Ruthel G, Buxbaum RE, Heidemann SR. Mechanical tension can specify axonal fate in hippocampal neurons. $\mathrm{J}$ Cell Biol. 2002;159:499-508.
International Journal of Nanomedicine

\section{Publish your work in this journal}

The International Journal of Nanomedicine is an international, peerreviewed journal focusing on the application of nanotechnology in diagnostics, therapeutics, and drug delivery systems throughout the biomedical field. This journal is indexed on PubMed Central, MedLine, CAS, SciSearch $®$, Current Contents $® /$ Clinical Medicine,

\section{Dovepress}

Journal Citation Reports/Science Edition, EMBase, Scopus and the Elsevier Bibliographic databases. The manuscript management system is completely online and includes a very quick and fair peer-review system, which is all easy to use. Visit http://www.dovepress.com/ testimonials.php to read real quotes from published authors.

Submit your manuscript here: http://www.dovepress.com/international-journal-of-nanomedicine-journal 